\section{ORIENTAÇÃO PARA ALIMENTAÇÃO SAUDÁVEL E FATORES ASSOCIADOS ENTRE USUÁRIOS DA ATENÇÃO PRIMÁRIA À SAÚDE NO SUL DO BRASIL}

\author{
Guidelines to healthy eating and associated factors among \\ users of primary health care in Southern Brazil \\ Orientación para alimentación saludable y factores asociados \\ entre usuarios de la atención primaria de salud del sur de \\ Brasil
}

\section{RESUMO}

Objetivo: Descrever a prevalência de orientação para alimentação saudável, diferenças entre modelo assistencial e fatores associados entre usuários da atenção primária de saúde. Métodos: Estudo transversal, realizado com 1.246 adultos e idosos, em Pelotas, Rio Grande do Sul. Os dados foram coletados entre maio e outubro de 2013, por meio da aplicação de questionário investigando dados socioeconômicos, doenças crônicas autorreferidas, autopercepção da saúde e da alimentação, estado nutricional, modelo assistencial em que é atendido e orientação para alimentação saudável. Aassociação entre as variáveis independentes e a orientação para alimentação saudável foi verificada por meio da Razão de Prevalências, comparando-se expostos e não expostos quanto à frequência da orientação. Resultados: A prevalência da orientação para alimentação saudável foi de 42\% (IC95 39,2-44,7) e apresentaram maior probabilidade: mulheres ( $\mathrm{RP}=1,51$; IC95 1,26-1,83), idosos $(\mathrm{RP}=1,39$; IC95 1,20-1,62), aqueles com maior número de doenças crônicas ( $\mathrm{RP}=1,62$; IC95 1,36-1,93), que autoperceberam sua alimentação como negativa ( $R P=1,32$; IC95 1,14-1,52) e atendidos pela Estratégia de Saúde da Família $(\mathrm{RP}=1,15 ; 1,02-1,30)$. A probabilidade da orientação para alimentação saudável mostrou-se menor entre os com cor da pele branca $(\mathrm{RP}=0,85$; IC95 0,74-0,97) e ensino médio e superior ou mais $(\mathrm{RP}=0,88$ e 0,83 respectivamente, $\mathrm{p}$ teste tendência linear $=0,037)$. Conclusão: $\mathrm{Na}$ atenção primária, a orientação para alimentação saudável não é universal e há iniquidade, deixando clara a necessidade de maiores esforços no sentido de ampliar a oferta. Maior atenção deve ser dada aos homens, aos indivíduos mais jovens, aos com cor da pele branca e àqueles sem diagnóstico de doenças crônicas.

Descritores: Avaliação em Saúde; Atenção Primária à Saúde; Educação Alimentar e Nutricional; Nutrição em Saúde Pública; Promoção da Saúde.

\section{ABSTRACT}

Objective: To describe the prevalence of guidelines to healthy eating, differences between health care models and associated factors among users of primary health care. Methods: Cross-sectional study conducted with 1,246 adults and older people in Pelotas, Rio Grande do Sul. Data were collected between May and October 2013 through a questionnaire to assess sociodemographic data, self-reported chronic disease, self-perception of health and nutrition, nutritional status, health care model used, and guidelines to healthy eating. The association between the independent variables and the guidelines to healthy eating was verified using Prevalence Ratio by comparing exposed and unexposed individuals according to the frequency of guidelines. Results: The prevalence of guidelines to healthy eating was 42\% (CI95 39.2-44.7) and women (PR 1.51; CI95 1.26-1.83), older people (PR 1.39; CI95 1.20-1.62), those with more chronic diseases (PR 1.62; CI95 1.36-1.93), those with a negative self-perception of nutrition (PR 1.32; CI95 1.14-1.52) and those treated within the Family Health Strategy (PR 1.15; 1.02-1.30) were more likely to be exposed to the guidelines. The probability of guidelines to healthy eating was lower among those with white skin color (PR 0.85; CI95 0.74-0.97) and secondary and higher education or more (PR 0.88 and 0.83 respectively, $p=0.037$ linear trend test). Conclusion: In primary care, the guidelines to healthy eating are not universal and there is iniquity, highlighting the need

Artigo Original

Ivana Loraine Lindemann ${ }^{(1,2)}$ Raúl Andres Mendoza-Sassi ${ }^{(1)}$

1) Universidade Federal do Rio Grande FURG - Rio Grande (RS) - Brasil

2) Universidade Federal da Fronteira Sul UFFS - Passo Fundo (RS) - Brasil

Recebido em: 08/12/2015

Revisado em: 17/02/2016 Aceito em: 15/03/2016 
for further efforts to increase its offer. Greater attention should be given to men, younger individuals, people with white skin color and those diagnosed with chronic diseases.

Descriptors: Health Evaluation; Primary Health Care; Food and Nutrition Education; Nutrition, Public Health; Health Promotion.

\section{RESUMEN}

Objetivo: Describir la prevalencia de orientación para alimentación saludable, las diferencias entre el modelo de asistencia y los factores asociados entre los usuarios de la atención primaria de salud. Métodos: Estudio transversal realizado con 1.246 adultos y mayores de Pelotas, Rio Grande do Sul. Los datos fueron recogidos entre mayo y octubre de 2013 a través de la aplicación de un cuestionario sobre los datos socioeconómicos, las enfermedades crónicas auto referidas, la auto percepción de la salud y la alimentación, el estado nutricional, el modelo de asistencia y la orientación para alimentación saludable. La asociación entre las variables independientes y la orientación para alimentación saludable fue verificada a través de la Razón de Prevalencias comparándose los expuestos y no expuestos cuanto a la frecuencia de la orientación. Resultados: La prevalencia de orientación para alimentación saludable fue del 42\% (IC95 39,244,7) y presentaron mayor probabilidad las mujeres $(R P=1,51$; IC95 1,26-1,83), los mayores ( $R P=1,39$; IC95 1,20-1,62), aquellos con más enfermedades crónicas ( $R P=1,62$; IC95 1,36-1,93), los que auto percibieron su alimentación como negativa $(R P=1,32$; IC95 1,14-1,52) y los asistidos por la Estrategia de Salud de la Familia $(R P=1,15 ; 1,02-1,30)$. La probabilidad de la orientación para alimentación saludable se mostró menor entre los que tenían el color de piel blanco $(R P=0,85$; IC95 0,74-0,97) y educación secundaria y superior o más $(R P=0,88$ e 0,83 respectivamente, $p$ prueba tendencia linear $=0,037)$. Conclusión: La orientación para alimentación saludable en la atención primaria no es universal y hay iniquidad lo que muestra la necesidad de más esfuerzos para la ampliación de la oferta. Más atención se debe dar a los hombres, a los individuos más jóvenes, a los de color de piel blanco y aquellos sin diagnóstico de enfermedades crónicas.

Descriptores: Evaluación en Salud; Atención Primaria de Salud; Educación Alimentaria y Nutricional; Nutrición en Salud Pública; Promoción de la Salud.

\section{INTRODUÇÃO}

Há evidências científicas que apontam para o papel fundamental da alimentação saudável na promoção da saúde, na prevenção de muitas doenças e no seu tratamento não farmacológico ${ }^{(1-3)}$. No Brasil, a orientação alimentar foi integrada ao campo de atuação do Sistema Único de Saúde (SUS) $)^{(4)}$, assinalando, de forma decisiva, a responsabilidade do Ministério da Saúde (MS) para com as condições alimentares e nutricionais da população brasileira.
As mudanças no perfil alimentar e nutricional da população e suas consequências no panorama de morbimortalidade têm repercutido nas políticas públicas de saúde. Diante disso, a promoção da alimentação saudável tem sido destacada, reiterando-se que cabe aos profissionais de saúde, especialmente aos que atuam na atenção primária, orientar a população visando à adoção de práticas alimentares saudáveis ${ }^{(5-7)}$.

Visando consolidar uma nova lógica de assistência, o MS adotou a Estratégia de Saúde da Família (ESF) como forma de reorientar o modelo assistencial, por meio da vinculação de equipes multiprofissionais a Unidades Básicas de Saúde (UBS) ${ }^{(8,9)}$, a fim de reorganizar a prática ${ }^{(10)}$ e priorizar o primeiro nível de assistência ${ }^{(9)}$, com ênfase na prevenção de doenças e na educação em saúde ${ }^{(11)}$.

As equipes multiprofissionais mínimas da ESF são compostas por médicos, enfermeiros, auxiliares ou técnicos de enfermagem e agentes comunitários da saúde ${ }^{(12)}$. Para apoiar e fortalecer a inserção da ESF, e com o intuito de ampliar e qualificar as ações da atenção primária, foram criados os Núcleos de Apoio à Saúde da Família (NASF) ${ }^{(10)}$, os quais caracterizam-se como equipes de profissionais de saúde, de diferentes áreas do conhecimento, que atuam em conjunto com as equipes mínimas, compartilhando e apoiando as práticas em saúde nos territórios sob sua responsabilidade. Os profissionais que integram os NASF são definidos pelos gestores municipais e equipes de ESF, mediante critérios de prioridades identificadas a partir das necessidades locais ${ }^{(13)}$.

Em um cenário ideal, as ações de promoção da alimentação saudável, as orientações e as terapias nutricionais específicas de acordo com os diagnósticos devem ser executadas pelo nutricionista, profissional habilitado para tal. Junto à ESF, o nutricionista pode compor o NASF, contribuindo com a qualificação e o fortalecimento das ações voltadas à alimentação saudável na atenção primária ${ }^{(10)}$.

Nos últimos anos, diferentes aspectos da atenção primária em saúde no Brasil têm sido estudados com bastante profundidade, abordando tanto a execução de programas voltados a grupos específicos quanto as diferenças de desempenho em termos de modelo tradicional e $\operatorname{ESF}^{(14,15)}$.

Entretanto, pouco se sabe sobre a ocorrência da orientação nesse ponto da rede de assistência, bem como sobre o efeito que a ESF tem sobre a mesma. De modo geral, a frequência e os fatores associados à orientação ainda não estão suficientemente esclarecidos, e os poucos trabalhos realizados constataram que essa ação ainda é incipiente. Apenas um terço da população adulta, residente em 100 cidades brasileiras, recebeu informações a respeito da ingestão de sal, açúcar ou gordura ${ }^{(16)}$. Dentre os usuários de uma UBS, em um município de grande porte, menos da metade recebeu orientação sobre alimentação saudável ${ }^{(17)}$. 
Com relação aos fatores associados a ter recebido orientação, figuram o sexo feminino, a idade avançada, a cor da pele branca, a maior renda ${ }^{(16)} \mathrm{e}$ o diagnóstico de doença crônica não transmissível (DCNT) $)^{(16,17)}$.

Assim, tendo em vista a importância da orientação dos usuários do SUS quanto à prática de uma alimentação adequada e saudável, bem como o papel da ESF nesse processo e a falta de pesquisas que tenham abordado especificamente este contexto, o objetivo deste estudo foi descrever a prevalência de orientação para alimentação saudável, diferenças entre modelo assistencial e fatores associados entre usuários da atenção primária de saúde.

\section{MÉTODOS}

Trata-se de um estudo transversal, realizado entre maio e outubro de 2013, na cidade de Pelotas/RS. A cidade, segundo o censo demográfico de 2010, tinha uma população estimada em 328.275 habitantes e Índice de Desenvolvimento Humano (IDH) de 0,739(18). Em 2013, a rede de atenção primária contava com 36 UBS na zona urbana, em 14 das quais estava implantada a ESF, indicando uma cobertura de $38,9 \%$, mas o município não tinha equipes de NASF.

Foram elegíveis usuários de todas as UBS urbanas, de ambos os sexos, com idade igual ou superior a 20 anos, excluídas mulheres em período de gestação e/ou lactação e portadores de deficiência física ou mental, em função da dieta e avaliação nutricional diferenciada ou dificuldade em responder o questionário.

O tamanho da amostra foi calculado considerando todas as variáveis independentes incluídas no estudo, risco relativo de 2,0 , nível de confiança de $95 \%$, poder de $80 \%$, relação de não expostos para expostos de até $1: 9$ e prevalência esperada do desfecho em não expostos de no mínimo 13\%. Seriam necessários 936 respondentes que, acrescidos de $10 \%$ para possíveis perdas e $25 \%$ para fatores de confusão, totalizaram 1.264. Incluíram-se todas as UBS da cidade com amostragem em duplo estágio. O primeiro foi aleatório proporcional, e o número médio de procedimentos realizados em cada UBS no mês anterior à coleta de dados serviu de critério para definir o número de usuários a ser entrevistado em cada UBS. O segundo foi de conveniência, e em cada UBS as duplas de entrevistadores entrevistavam de forma consecutiva os usuários presentes para consulta. Não se atingindo o número definido para a UBS, os entrevistadores retornavam no dia seguinte, e assim consecutivamente, até completar o número estipulado, sem repetição de usuários.

Aferiu-se o desfecho por meio da seguinte pergunta: "Alguma vez, durante uma consulta, um médico ou outro profissional de saúde o orientou a ter uma alimentação saudável?". Àqueles que responderam afirmativamente, perguntou-se o local da consulta e consideraram-se com desfecho positivo os participantes orientados em UBS.

As variáveis independentes analisadas contemplaram sexo, idade (medida em anos completos e categorizada em 20-39, 40-59 e 60 ou mais), cor da pele autorreferida (branca, preta e outras), situação conjugal (com cônjuge, sem cônjuge), quintis de renda familiar mensal per capita (sendo o $1^{\circ}$ o menos favorecido economicamente), escolaridade (Ensino Fundamental, Médio e Superior ou mais), ocupação (trabalha, não trabalha), número de doenças crônicas não transmissíveis (DCNT) autorreferidas (referência ao diagnóstico médico de obesidade, diabetes, hipertensão arterial, hipercolesterolemia, hipertrigliceridemia ou doença cardíaca, categorizadas em nenhuma, 1-2 e 3 ou mais), autopercepção da saúde e da alimentação (positiva, negativa), modelo assistencial implantado na UBS em que o usuário estava sendo atendido (tradicional, Estratégia de Saúde da Família) e estado nutricional. O estado nutricional foi avaliado pelo peso e altura autorreferidos e classificado pelo Índice de Massa Corporal ${ }^{(5)}$. Para fins de análise, categorizou-se o estado nutricional em eutrofia ou excesso de peso.

As entrevistas, com um questionário pré-codificado e testado, foram realizadas por 12 entrevistadores treinados que, em duplas, apresentavam-se à recepção das UBS, buscavam os sujeitos elegíveis para o estudo e os convidavam a responder ao questionário, mediante consentimento livre e esclarecido. Havendo recusa, eram feitas duas novas tentativas, pelo outro entrevistador da dupla e pelo supervisor de campo, e não houve reposição de perdas. As coletas ocorreram de segunda a sexta-feira, nos turnos manhã e tarde, na sala de espera, antes das consultas, até que se completasse o número estipulado para cada unidade. De todas as entrevistas realizadas, $10 \%$ foram refeitas para o controle de qualidade.

Os dados foram duplamente digitados no programa EpiData 3.1, e a análise estatística, realizada no pacote estatístico Stata, versão 11, compreendeu a descrição da amostra e o cálculo da prevalência do desfecho e seu intervalo de confiança de 95\% (IC95). Posteriormente, fezse a análise bivariada para verificar as associações entre o desfecho e os fatores de risco, sendo calculadas as Razões de Prevalências (RP) brutas e seus IC95.

Em seguida, na análise multivariada, utilizou-se a Regressão de Poisson com variância robusta, considerando a opção para amostra por conglomerados do Stata (robust). A análise do tipo backward stepwise, seguiu um modelo hierárquico ${ }^{(19)}$ pré-estabelecido, o qual compreendeu três níveis de determinação, sendo calculadas as RP ajustadas e seus IC95. O nível mais distal incluiu as variáveis demográficas e socioeconômicas (sexo, idade, cor da pele, situação conjugal, renda, escolaridade e ocupação); no segundo, incluíram-se as variáveis referentes às condições 
de saúde e de alimentação (número de DCNT autorreferidas, autopercepção da saúde, estado nutricional e autopercepção da alimentação); e no terceiro, o modelo assistencial. Todas as variáveis de cada nível eram ajustadas entre si e com as variáveis do nível subsequente. Aquelas variáveis com um $p$ menor ou igual a 0,20 eram mantidas para ajuste com o nível seguinte, de forma a evitar a possibilidade de confusão negativa. No caso de variáveis categóricas politômicas, quando houve ordenamento entre elas, foi realizado o teste de Wald para tendência linear, e quando não houve ordenamento evidente, o teste de heterogeneidade. Em todos os testes de significância, considerou-se um valor de $p<0,05$ de um teste bicaudal.

O Comitê de Ética em Pesquisa na Área da Saúde da instituição aprovou o protocolo do estudo, parecer $\mathrm{n}^{\circ}$ 228.401, obedecendo à Resolução $\mathrm{n}^{\circ} 466 / 12$ do Conselho Nacional de Saúde e aos princípios éticos contidos na Declaração de Helsinki.

\section{RESULTADOS}

Dos 1.264 usuários arrolados, 1.246 responderam ao questionário, totalizando $1,4 \%$ de perdas $(\mathrm{n}=18)$. A maioria

Tabela I - Caracterização de usuários da rede urbana de Atenção Primária de Saúde. Pelotas, RS, 2013. (n=1.246).

\begin{tabular}{|c|c|c|}
\hline \multirow{2}{*}{\multicolumn{3}{|c|}{$\begin{array}{l}\text { Variáveis } \\
\text { Demográficas }\end{array}$}} \\
\hline & & \\
\hline \multicolumn{3}{|l|}{ Sexo } \\
\hline Masculino & 203 & 16,3 \\
\hline Feminino & 1.043 & 83,7 \\
\hline \multicolumn{3}{|l|}{ Idade em anos completos } \\
\hline $20-39$ & 471 & 37,8 \\
\hline $40-59$ & 498 & 40,0 \\
\hline 60 ou mais & 277 & 22,2 \\
\hline \multicolumn{3}{|l|}{ Cor da pele* } \\
\hline Preta e outras & 457 & 36,7 \\
\hline Branca & 787 & 63,3 \\
\hline \multicolumn{3}{|l|}{ Situação conjugal } \\
\hline Sem cônjuge & 496 & 39,8 \\
\hline Com cônjuge & 750 & 60,2 \\
\hline \multicolumn{3}{|l|}{ Socioeconômicas } \\
\hline Quintis de renda familiar mensal per capita & Média & DP \\
\hline $1^{\circ}$ & 142,9 & 71,7 \\
\hline $2^{\circ}$ & 289,5 & 37,9 \\
\hline $3^{\circ}$ & 416,8 & 51,8 \\
\hline $4^{\mathrm{o}}$ & 629,1 & 67,1 \\
\hline $5^{\circ}$ & $1.034,8$ & 304,8 \\
\hline \multicolumn{3}{|l|}{ Escolaridade } \\
\hline Ensino Fundamental & 832 & 66,8 \\
\hline Ensino Médio & 276 & 22,1 \\
\hline Ensino Superior ou mais & 138 & 11,1 \\
\hline \multicolumn{3}{|l|}{ Ocupação } \\
\hline Trabalha & 396 & 31,8 \\
\hline Não trabalha & 850 & 68,2 \\
\hline \multicolumn{3}{|l|}{ Condições de saúde e de alimentação } \\
\hline \multicolumn{3}{|c|}{ Número de doenças crônicas não transmissíveis autorreferidas } \\
\hline Nenhuma & 576 & 46,2 \\
\hline $1-2$ & 486 & 39,0 \\
\hline 3 ou mais & 184 & 14,8 \\
\hline \multicolumn{3}{|l|}{ Autopercepção da saúde } \\
\hline Positiva & 728 & 58,4 \\
\hline Negativa & 518 & 41,6 \\
\hline \multicolumn{3}{|l|}{ Estado nutricional $* *$} \\
\hline Eutrofia & 434 & 39,0 \\
\hline Excesso de peso & 679 & 61,0 \\
\hline \multicolumn{3}{|l|}{ Autopercepcão da alimentação } \\
\hline Positiva & 787 & 63,2 \\
\hline Negativa & 459 & 36,8 \\
\hline \multicolumn{3}{|l|}{ Modelo assistencial } \\
\hline Tradicional & 577 & 46,3 \\
\hline Estratégia de Saúde da Família & 669 & 53,7 \\
\hline
\end{tabular}

* 2 perdas; ** 133 perdas. 
era do sexo feminino $(83,7 \%, \mathrm{n}=1.043)$, adulta $(77,8 \%$, $\mathrm{n}=969)$, tinha cor da pele branca $(63,3 \%, \mathrm{n}=787)$, cônjuge $(60,2 \%, n=750)$, ensino fundamental completo $(66,8 \%$, $\mathrm{n}=832)$ e não estava trabalhando $(68,2 \%, \mathrm{n}=850)$. A renda per capita variou de $\mathrm{R} \$ 0,0$ a $\mathrm{R} \$ 3.333,3$ (média 496,9 $\pm \mathrm{DP}$ 344,1) (Tabela I).
Sobre as condições de saúde e de alimentação, a maior parte referiu diagnóstico médico de uma ou mais DCNT $(53,8 \%, n=670)$, tinha autopercepção positiva da saúde $(58,4 \%, n=728)$ e da alimentação $(63,2 \%, n=787)$ e excesso de peso $(61 \%, n=679)$. No que se refere ao modelo assistencial, 53,7\% $(\mathrm{n}=669)$ eram usuários da $\mathrm{ESF}$. (Tabela I).

Tabela II - Análise bruta e ajustada de fatores associados à orientação para alimentação saudável, referida por usuários da rede urbana de Atenção Primária de Saúde. Pelotas, RS, 2013. (n=1.246).

\begin{tabular}{|c|c|c|c|c|}
\hline \multirow{2}{*}{ Variáveis } & \multicolumn{2}{|l|}{ Bruta } & \multicolumn{2}{|c|}{ Ajustada } \\
\hline & RP (IC95) & Valor de $p$ & RP (IC95) & Valor de $\mathrm{p}$ \\
\hline \multicolumn{5}{|l|}{$1^{0}$ nível* $^{*}$} \\
\hline \multicolumn{5}{|l|}{ Demográficas } \\
\hline Sexo & & $<0,001^{\text {a }}$ & & $<0,001^{\mathrm{a}}$ \\
\hline Feminino & $1,42(1,18-1,71)$ & & $1,51(1,26-1,83)$ & \\
\hline Idade em anos completos & & $<0,001^{\mathrm{b}}$ & & $<0,001^{\mathrm{b}}$ \\
\hline $40-59$ & $1,31(1,17-1,48)$ & & $1,33(1,17-1,51)$ & \\
\hline 60 ou mais & $1,36(1,18-1,56)$ & & $1,39(1,20-1,62)$ & \\
\hline Cor da pele & & $0,098^{\mathrm{a}}$ & & $0,016^{\mathrm{a}}$ \\
\hline Branca & $0,89(0,78-1,02)$ & & $0,85(0,74-0,97)$ & \\
\hline Situação conjugal & & $0,234^{\mathrm{a}}$ & & $0,948^{\mathrm{a}}$ \\
\hline Com cônjuge & $0,94(0,85-1,04)$ & & $1,00(0,89-1,12)$ & \\
\hline \multicolumn{5}{|l|}{ Socioeconômicas } \\
\hline Quintis de renda familiar mensal per capita & & $0,068^{\mathrm{c}}$ & & $0,092^{\mathrm{c}}$ \\
\hline $2^{\mathrm{o}}$ & $1,09(0,93-1,27)$ & & $1,08(0,93-1,26)$ & \\
\hline $3^{\circ}$ & $0,90(0,72-1,11)$ & & $0,91(0,74-1,11)$ & \\
\hline $4^{\circ}$ & $1,04(0,88-1,23)$ & & $1,02(0,85-1,22)$ & \\
\hline $5^{\circ}$ & $1,05(0,91-1,21)$ & & $1,09(0,93-1,26)$ & \\
\hline Escolaridade & & $0,002^{\mathrm{b}}$ & & $0,037^{\mathrm{b}}$ \\
\hline Ensino Médio & $0,83(0,72-0,96)$ & & $0,88(0,76-1,02)$ & \\
\hline Ensino Superior ou mais & $0,81(0,68-0,97)$ & & $0,83(0,68-1,03)$ & \\
\hline Ocupação & & $0,047^{\mathrm{a}}$ & & $0,498^{\mathrm{a}}$ \\
\hline Não trabalha & $1,15(1,00-1,32)$ & & $1,05(0,91-1,22)$ & \\
\hline \multicolumn{5}{|l|}{$2^{\mathbf{0}}$ nível** } \\
\hline \multicolumn{5}{|l|}{ Condições de saúde e de alimentação } \\
\hline Número de DCNT autorreferidas & & $<0,001^{\mathrm{b}}$ & & $<0,001^{\mathrm{b}}$ \\
\hline $1-2$ & $1,51(1,30-1,75)$ & & $1,40(1,19-1,64)$ & \\
\hline 3 ou mais & $1,94(1,63-2,30)$ & & $1,62(1,36-1,93)$ & \\
\hline Autopercepção da saúde & & $<0,001^{\mathrm{a}}$ & & $0,531^{\mathrm{a}}$ \\
\hline Negativa & $1,33(1,15-1,53)$ & & $1,05(0,89-1,24)$ & \\
\hline Estado nutricional & & $<0,001^{\text {a }}$ & & $0,057^{\mathrm{a}}$ \\
\hline Excesso de peso & $1,27(1,12-1,44)$ & & $1,16(1,00-1,35)$ & \\
\hline Autopercepção da alimentação & & $<0,001^{\text {a }}$ & & $<0,001^{\mathrm{a}}$ \\
\hline Negativa & $1,43(1,25-1,65)$ & & $1,32(1,14-1,52)$ & \\
\hline $3^{\circ}$ nível** & & & & \\
\hline Modelo assistencial & & $0,026^{\mathrm{a}}$ & & $0,021^{\mathrm{a}}$ \\
\hline Estratégia de Saúde da Família & $1,15(1,02-1,30)$ & & $1,15(1,02-1,30)$ & \\
\hline
\end{tabular}

DCNT: doenças crônicas não transmissíveis; RP: Razão de Prevalência; IC95: Intervalo de Confiança de 95\%. Testes: a) Teste do Quiquadrado, b) teste de tendência linear, c) teste de heterogeneidade; Perdas: *2 perdas, **134 perdas; Categorias de referência: sexo masculino, 20-39 anos de idade, cor da pele preta e outras, sem cônjuge, $1^{\circ}$ quintil de renda, ensino fundamental, trabalha, nenhuma DCNT autorreferida, autopercepção positiva da saúde, eutrofia, autopercepção positiva da alimentação, modelo de atenção à saúde tradicional. 
Dos entrevistados 42\% ( $\mathrm{n}=523$; IC95 39,2-44,7) referiram orientação para alimentação saudável. Quando as variáveis independentes foram ajustadas seguindo o modelo hierárquico pré-estabelecido, no primeiro nível, o sexo feminino manteve a associação com o desfecho. As mulheres tiveram uma probabilidade de orientação $51 \%$ superior aos homens ( $\mathrm{RP}=1,51$; IC95 1,26-1,83). (Tabela II).

A idade manteve a tendência linear de associação com o desfecho, tendo os idosos uma probabilidade $39 \%$ maior de serem orientados em comparação àqueles entre 20 e 39 anos de idade ( $\mathrm{RP}=1,39$; IC95 1,20-1,62). A cor da pele passou a associar-se de forma significativa, sendo que os de cor branca tiveram probabilidade $15 \%$ menor de serem orientados ( $\mathrm{RP}=0,85$; IC95 0,74-0,97). Percebeu-se ainda que quanto maior a escolaridade menor a probabilidade do desfecho, com redução de $12 \%$ entre usuários com ensino médio e $17 \%$ entre aqueles com ensino superior ou mais ( $\mathrm{RP}=0,88$; IC95 0,76-1,02; RP=0,83; IC95 0,68-1,03; $p$ de tendência linear $=0,037)$, e que o efeito da ocupação se perdeu com o ajuste (RP=1,05; IC95 0,91-1,22) (Tabela II).

No segundo nível, manteve-se a tendência linear em relação ao número de DCNT autorreferidas, sendo que aqueles com diagnóstico médico de três ou mais doenças, mostraram probabilidade de desfecho $62 \%$ maior $(\mathrm{RP}=1,62$; IC95 1,36-1,93). A probabilidade de orientação mostrou-se $32 \%$ maior naqueles que autoperceberam sua alimentação como negativa ( $\mathrm{RP}=1,32$; IC95 1,14-1,52), e o efeito da autopercepção da saúde e do estado nutricional se perdeu após ajuste. Ainda, os usuários atendidos pela ESF apresentaram probabilidade $15 \%$ maior de receberem orientação para alimentação saudável $(\mathrm{RP}=1,15 ; 1,02-1,30)$ (Tabela II).

\section{DISCUSSÃO}

Apesar da reconhecida importância da alimentação saudável e da vasta recomendação, especialmente em termos de sistema público de saúde, para que a população seja orientada em relação a práticas alimentares adequadas e saudáveis, faltam estudos que abordem, de uma forma representativa, a oferta desta ação na atenção primária em saúde no Brasil. Assim, esta pesquisa foi realizada em toda a rede urbana de atenção primária de um município de porte médio na região Sul do país e mostrou que menos da metade dos usuários recebeu a referida orientação.

Os resultados apontaram uma prevalência de orientação para alimentação saudável de $42 \%$, superior, portanto, às encontradas em estudos de base populacional nos Estados Unidos e no Canadá, respectivamente, de $21,3 \%^{(20)}$ e de $37,6 \%{ }^{(1)}$. O valor encontrado é também superior aos $20,3 \%$ mencionados em outro estudo brasileiro, realizado com usuários de uma UBS com ESF, em Belo Horizonte, estado de Minas Gerais ${ }^{(17)}$. Da mesma forma, ainda que o desfecho seja distinto, os achados superam os de um estudo de base populacional no Brasil, o qual apontou prevalências em torno de $30 \%$ para orientações alimentares específicas ${ }^{(16)}$. É provável que os resultados tenham sido diferentes em função da distinção entre as amostras, não sendo a do presente estudo de base populacional, tendo, porém, incluído usuários de toda a rede de atenção primária da cidade.

No tocante às características demográficas e socioeconômicas, mesmo após o ajuste, manteve-se o efeito do sexo feminino sobre a ocorrência do desfecho, diferentemente do que foi verificado nos Estados Unidos, onde as variáveis não mostraram associação entre $\mathrm{si}^{(20)}$, e no Canadá, onde houve maior probabilidade de orientação entre os homens ${ }^{(1)}$. Contudo, o resultado encontrado está em consonância com outras pesquisas nacionais ${ }^{(16,17)}$, evidenciando que, de fato, no Brasil, as mulheres têm maior probabilidade de orientação para uma alimentação saudável. Isso pode ser devido a elas utilizarem mais os serviços de saúde em comparação aos homens ${ }^{(21)}$, além de serem, na maioria das vezes, responsáveis pela alimentação da família. Sabe-se ainda que as mulheres são mais preocupadas com questões tanto de saúde como de dieta, especialmente associadas à estética ${ }^{(22-24)}$, o que pode leválas a inquirirem mais a respeito de cuidados no momento da consulta.

Quanto maior a idade, maior a probabilidade de receber orientação, o que se distingue da pesquisa americana, em que não se constataram diferenças ${ }^{(20)}$, e da canadense, em que a probabilidade de orientação foi maior nos indivíduos entre 35 e 54 anos $^{(1)}$. Porém, os resultados da presente pesquisa corroboram com outros que, embora sejam referentes apenas a adultos, apresentaram, de maneira similar, uma tendência linear de aumento na probabilidade de orientação conforme acréscimo da idade ${ }^{(16)}$. A razão para isso pode residir no aumento da prevalência de doenças crônicas ao longo da vida, cujas recomendações específicas de alimentação integram o tratamento, bem como no fato de que os idosos são mais preocupados com saúde e alimentação em relação aos mais jovens, o que pode levar a uma maior frequência de orientação ${ }^{(25-27)}$.

Perceberam-se diferenças em relação à cor da pele, o que distingue os resultados da pesquisa feita com população americana. Porém, estudo realizado no Brasil ${ }^{(16)}$ também identificou maior orientação para ingestão de pouco sal e pouca gordura entre brasileiros com cor da pele preta, achados esses que podem estar relacionados à associação entre cor da pele e hipertensão arterial ${ }^{(28)}$. 
No que se refere às condições de saúde e de alimentação, de forma consistente com a literatura ${ }^{(1,16,17)}$, os resultados apontaram que quanto maior o número DCNT autorreferidas, maior a probabilidade de orientação para alimentação saudável. Contudo, embora outros pesquisadores tenham verificado associação entre o excesso de peso e maior probabilidade de orientação alimentar ${ }^{(16,20)}$, nos resultados deste estudo o efeito dessa variável se perdeu com o ajuste, possivelmente devido à associação do desfecho com DCNT autorreferidas.

A maior probabilidade do desfecho entre aqueles com doença crônica era acreditada, pois, tendo em vista que a alimentação compõe o tratamento não farmacológico dessas enfermidades, é provável que, conforme o recomendado, muitos usuários tenham recebido orientações alimentares a partir do diagnóstico. Por outro lado, se as DCNT estão entre as principais causas de morbimortalidade no país e são responsáveis por $58 \%$ dos anos de vida perdidos precocemente $^{(7)}$, a observação de que mais da metade dos entrevistados tinha pelo menos uma DCNT pode estar indicando um enfoque das ações nos serviços de saúde ainda com caráter mais curativo do que preventivo e de promoção da saúde.

A alimentação é um dos principais fatores associados às DCNT, e a sua promoção, de forma saudável, está inserida como eixo temático na Política Nacional de Promoção da Saúde ${ }^{(6)}$ e figura como diretriz da Política Nacional de Alimentação e Nutrição( ${ }^{(7)}$, visando, além do cuidado integral aos agravos já instalados, a realização de ações de prevenção em todas as fases da vida para diminuir a sua ocorrência. Entretanto, a elevada prevalência dessas doenças, tanto na população em geral como também entre os usuários do sistema público de saúde, sugere a existência de falhas, cuja elucidação requer um aprofundamento por meio de estudos que abarquem questões relacionadas à cobertura populacional pela ESF; à oferta de serviços baseada em demanda espontânea; à formação, capacitação e condições de trabalho dos profissionais de saúde vinculados à atenção primária; e ao aspecto intersetorial da promoção da saúde e da alimentação saudável.

Ainda dentre os fatores analisados neste estudo, os usuários que perceberam sua alimentação como negativa apresentaram maior probabilidade de orientação, sugerindo que, ao julgar sua alimentação inadequada, o usuário demande mais atenção dos profissionais de saúde. O efeito da autopercepção da saúde se perdeu com o ajuste, e os dados disponíveis na literatura não demonstram consistência em relação ao efeito deste fator ${ }^{(1,20)}$.

Quanto ao modelo assistencial, inicialmente, é importante esclarecer que mesmo sendo a cobertura da ESF na rede urbana de $38,9 \%$, a maioria dos entrevistados utilizou a ESF (53,7\%), e essa diferença é devido à maior proporção de atendimentos em unidades com a estratégia. Os resultados demonstraram que, conforme o esperado devido ao caráter preventivo e educativo da $\operatorname{ESF}^{(11)}$, a probabilidade do desfecho foi maior entre os usuários atendidos por esse modelo, embora este não tenha sido verificado em relação ao aconselhamento para prática de atividade física na atenção primária ${ }^{(29)}$.

Entretanto, é necessário destacar que menos da metade dos usuários recebeu orientação para alimentação saudável, indicando que, além dos fatores aqui estudados, os quais apontaram para dificuldades na orientação vinculadas a aspectos sociodemográficos e a condições de saúde e de alimentação dos usuários, outras barreiras devem estar dificultando o cumprimento dessa tarefa.

Cabe frisar que, neste estudo, não se objetivou avaliar qual profissional fez a orientação, a natureza do seu conteúdo, bem como o entendimento e a adesão por parte do usuário, ou ainda o impacto em sua qualidade de vida. $\mathrm{O}$ intuito foi verificar se a ação recomendada pelas políticas e programas de saúde de fato chega ao usuário do sistema. Informações adicionais, úteis à necessária ampliação da oferta, poderiam ser obtidas por meio de uma análise a partir da visão dos profissionais, envolvendo desde a frequência da oferta da orientação, até sua capacitação para a ação, seja esta oriunda da própria formação profissional, da busca ativa por conhecimento e qualificação ou de ações de educação permanente oferecidas por parte da gestão do sistema de saúde.

Outros pontos a serem elucidados envolvem a qualidade e a eficácia da orientação, quando ela ocorre, bem como a atuação das equipes de NASF, no sentido de avaliar se em municípios em que a proposta está implantada, a realidade se apresenta de forma semelhante ou distinta da encontrada nesta pesquisa. Com a inclusão de nutricionistas trabalhando de forma matricial com as equipes, estimulando a orientação e contribuindo com a educação permanente, esperaria-se uma maior prevalência de orientação, pois os profissionais poderiam sentir-se mais seguros para a execução das orientações, tendo em vista que a falta de conhecimentos é, segundo eles, um dos principais motivos para a baixa oferta ${ }^{(30)}$.

Antes de concluir, faz-se necessário considerar as limitações que podem ter influenciado os achados deste estudo. Cabe salientar que, devido à natureza transversal do delineamento, os fatores apontados não podem ser considerados determinantes e sim, associados ao desfecho. Outra limitação é a possibilidade de causalidade reversa entre determinados fatores e o desfecho estudado. Contudo, ambas eram esperadas e são inerentes ao estudo transversal que é o mais utilizado para avaliar serviços de saúde. $\mathrm{Na}$ aferição do desfecho, devido à intenção de se conhecer a ocorrência da orientação ao longo do contato do usuário 
com o serviço, não se estipulou um período de tempo determinado, o que pode ter subestimado a prevalência pela possibilidade de viés de memória. Por ser um estudo de base de serviços de saúde, incluindo usuários da atenção primária, a amostra apresentou uma maior proporção de mulheres e, assim, os resultados não são generalizáveis a adultos e idosos. Além disso, a realização das entrevistas na sala de espera das UBS pode ter interferido nos resultados, sub ou super estimando algumas variáveis. Em contrapartida, destacam-se como pontos fortes da pesquisa o baixo percentual de perdas e o poder estatístico adequado para a maior parte das análises realizadas.

\section{CONCLUSÃO}

$\mathrm{Na}$ atenção primária, a orientação para alimentação saudável não é universal e há iniquidade, deixando clara a necessidade de maiores esforços no sentido de ampliar a oferta. Maior atenção deve ser dada aos homens, aos indivíduos mais jovens, aos com cor da pele branca e àqueles sem diagnóstico de doenças crônicas.

\section{REFERÊNCIAS}

1. Sinclair J, Lawson B, Burge F. Which patients receive advice on diet and exercise? Do certain characteristics affect whether they receive such advice? Can Fam Physician. 2008;54(3):404-12.

2. Jaime PC, Silva ACF, Lima AMC, Bortolini GA. Ações de alimentação e nutrição na atenção básica: a experiência de organização no Governo Brasileiro. Rev Nutr. 2011;24(6):809-24.

3. American Diabetes Association. Standards of Medical Care in Diabetes 2014. Diabetes Care. 2014;37(Suppl 1):14-80.

4. BRASIL. Lei $\mathrm{n}^{\circ}$ 8.080, de 19 de setembro de 1990. Dispõe sobre as condições para a promoção, proteção e recuperação da saúde, a organização e o funcionamento dos serviços correspondentes e dá outras providências. Diário Oficial da União, 20 set 1999. Disponível em: http://www.planalto.gov.br/ccivil_03/leis/L8080.htm

5. Ministério da Saúde (BR), Secretaria de Atenção à Saúde, Departamento de Atenção Básica, Coordenação Geral de Alimentação e Nutrição. Política Nacional de Alimentação e Nutrição: SISVAN na assistência à saúde. Brasília: Ministério da Saúde; 2008 [acesso em 2012 Jan 23]. Disponível em: http://189.28.128.100/ nutricao/docs/geral/protocolo_sisvan.pdf

6. Ministério da Saúde (BR), Secretaria de Vigilância em Saúde, Secretaria de Atenção à Saúde. Política Nacional de promoção da saúde. $3^{\mathrm{a}}$ ed. Brasília: Ministério da
Saúde; 2010. (Série B. Textos Básicos de Saúde. Série Pactos pela Saúde, n ${ }^{\circ}$ 7).

7. Ministério da Saúde (BR), Secretaria de Atenção à Saúde, Departamento de Atenção Básica. Política Nacional de Alimentação e Nutrição. Brasília: Ministério da Saúde; 2012. (Série B. Textos Básicos de Saúde).

8. Marques RM, Mendes A. Atenção Básica e Programa de Saúde da Família (PSF): novos rumos para a política de saúde e seu financiamento? Ciênc Saúde Coletiva. 2003;8(2):403-15.

9. Ministério da Saúde (BR), Secretaria de Atenção à Saúde, Departamento de Atenção Básica. Política Nacional de Atenção Básica. Brasília: Ministério da Saúde; 2012.

10. Ministério da Saúde (BR), Secretaria de Atenção à Saúde, Departamento de Atenção Básica. Diretrizes do Núcleo de Apoio à Saúde da Família. Brasília: Ministério da Saúde; 2009. (Série A. Normas e Manuais Técnicos, Cadernos de Atenção Básica, no 27).

11. Goldbaum M, Gianini RJ, Novaes HMD, César CLG. Health services utilization in areas covered by the family health program (Qualis) in Sao Paulo City, Brazil. Rev Saúde Pública. 2005;39(1):90-9

12. Ministério da Saúde (BR). Portaria no 2.488. Aprova a Política Nacional de Atenção Básica, estabelecendo a revisão de diretrizes e normas para a organização da Atenção Básica, para a Estratégia Saúde da Família (ESF) e o Programa de Agentes Comunitários de Saúde (PACS) [acesso em 2014 Jun 01]. Disponível em: http://www.saude.mt.gov.br/upload/legislacao/2488\%5B5046-041111-SES-MT\%5D.pdf

13. Ministério da Saúde (BR), Secretaria de Atenção à Saúde, Departamento de Atenção Básica. Diretrizes do Núcleo de Apoio à Saúde da Família. Volume 1: Ferramentas para a gestão e para o trabalho cotidiano. Brasília: Ministério da Saúde; 2014. (Cadernos de Atenção Básica, no 39).

14. Mendoza-Sassi RA, Cesar JA, Teixeira TP, Ravache C, Araújo GD, Silva TC. Diferenças no processo de atenção ao pré-natal entre unidades da Estratégia Saúde da Família e unidades tradicionais em um município da Região Sul do Brasil. Cad Saúde Pública. 2011;27(4):787-96.

15. Cesar JA, Sutil AT, Santos GB, Cunha CF, MendozaSassi RA. Assistência pré-natal nos serviços públicos e privados de saúde: estudo transversal de base populacional em Rio Grande, Rio Grande do Sul, Brasil. Cad Saúde Pública. 2012;28(11):2106-14. 
16. Silva SM, Facchini LA, Tomasi E, Piccini R, Thumé E, Silveira DS et al. Recebimento de orientação sobre consumo de sal, açúcar e gorduras em adultos: um estudo de base nacional. Rev Bras Epidemiol. 2013;16(4):995-1004.

17. Santos RP, Horta PM, Souza CS, Santos CA, Oliveira HBS, Almeida LMR, et al. Aconselhamento sobre alimentação e atividade física: prática e adesão de usuários da atenção primária. Rev Gaúch Enferm. 2012;33(4):14-21.

18. Instituto Brasileiro de Geografia e Estatística. Índice de Desenvolvimento Humano Municipal - 2010 (IDHM 2010). [acesso em 2014 Maio 10]. Disponível em: http:/www.cidades.ibge.gov.br/xtras/temas.php?lang $=\&$ codmun $=431440 \&$ idtema $=16 \&$ search=rio-grandedo-sul|pelotas|sintese-das-informacoes

19. Victora CG, Huttly SR, Fuchs SC, Olinto MTA. The role of conceptual frameworks in epidemiological analysis: a hierarchical approach. Int J Epidemiol. 1997;26(1):224-7.

20. Honda K. Factors underlying variation in receipt of physician advice on diet and exercise: applications of the behavioral model of health care utilization. Am J Health Promot. 2004;18(5):370-7.

21. Travassos C, Viacava F, Pinheiro R, Brito A. Utilização dos serviços de saúde no Brasil: gênero, características familiares e condição social. Rev Panam Salud Publica. 2002;11(5/6):365-73.

22. Beydoun MA, Wang Y. Do nutrition knowledge and beliefs modify the association of socio-economic factors and diet quality among US adults? Prev Med. 2008;46(2):145-53.

23. Vinholes DB, Assunção MCF, Neutzling MB. Frequência de hábitos saudáveis de alimentação medidos a partir dos 10 Passos da Alimentação Saudável do Ministério da Saúde. Pelotas, Rio Grande do Sul, Brasil. Cad Saúde Pública. 2009;25(4):791-9.

24. Zart VB, Aerts D, Rosa C, Béria JU, Raymann BW, Gigante LP et al. Cuidados alimentares e fatores associados em Canoas, RS, Brasil. Epidemiol Serv Saúde. 2010;19(2):143-54.
25. Estaquio C, Druesne-Pecollo N, Latino-Martel $\mathrm{P}$, Dauchet L, Hercberg S, Bertrais S. Socioeconomic differences in fruit and vegetable consumption among middle-aged french adults: adherence to the 5 a Day recommendation. JAm DietAssoc. 2008;108(12):202130 .

26. Instituto Brasileiro de Geografia e Estatística. Pesquisa de Orçamentos Familiares (POF) 2008-2009: análise do consumo alimentar pessoal no Brasil. Rio de Janeiro: IBGE; 2011. [acessado 2011 Out 12]. Disponível em: http://www.ibge.gov.br/home/estatistica/populacao/ condicaodevida/pof/2008_2009_encaa/default.shtm

27. Ministério da Saúde (BR), Secretaria de Vigilância em Saúde, Secretaria de Gestão Estratégica e Participativa. VIGITEL Brasil 2010: vigilância de fatores de risco e proteção para doenças crônicas por inquérito telefônico. Brasília: Ministério da Saúde; 2011.

28. Costa JSD, Barcellos FC, Sclowitz ML, Sclowitz IKT, Castanheira M, Olinto MTA et al. Prevalência de hipertensão arterial em adultos e fatores associados: um estudo de base populacional urbana em Pelotas, Rio Grande do Sul, Brasil. Arq Bras Cardiol. 2007;88(1):5965.

29. Siqueira FV, Nahas MV, Facchini LA, Silveira DS, Piccini RX, Tomasi E, et al. Aconselhamento para a prática de atividade física como estratégia de educação à saúde. Cad Saúde Pública. 2009;25(1):203-13.

30. Pimentel VRM, Sousa MF, Hamann EM, Mendonça AVM. Alimentação e nutrição na Estratégia Saúde da Família em cinco municípios brasileiros. Ciênc Saúde Coletiva. 2014;19(1):49-57.

\section{Endereço para correspondência:}

Ivana Loraine Lindemann

Curso de Medicina da UFFS

Rodovia RS 153, Km 03

CEP 99034-600 - Passo Fundo - RS - Brasil

E-mail: ivanaloraine@hotmail.com 